\title{
DESIGN TRADE-OFFS OF MICROMACHINED GYROSCOPE CONCEPT ALLOWING INTERCHANGEABLE OPERATION IN BOTH ROBUST AND PRECISION MODES
}

\author{
A.R. Schofield*, A.A. Trusov, and A.M. Shkel \\ MicroSystems Laboratory, Department of Mechanical and Aerospace Engineering, \\ University of California, Irvine, CA, USA
}

\begin{abstract}
This paper presents design trade-offs for a z-axis gyroscope allowing interchangeable operation in either precision or robust modes. This is enabled through a complete 2-DOF sense mode which allows the specification of the peak spacing and degree of coupling independent of frequency. Simulated sense mode frequency responses for various coupling amounts indicate that weakly coupled systems are desirable due to the larger precision mode gain increase (over $20 \mathrm{~dB}$ ) for atmospheric pressure operation. Experimentally measured sense mode frequency responses for strong and weak coupling confirm the simulation results in air while also revealing that strong coupling is desirable at vacuum due to the increased precision mode gain improvement of over $40 \mathrm{~dB}$ while leaving the robust mode relatively unaffected.
\end{abstract}

\section{KEYWORDS}

Vibratory gyroscope, Robustness, Mode-matching

\section{INTRODUCTION}

Micromachined vibratory gyroscopes operate based on the Coriolis effect where a transfer of energy occurs between the orthogonal drive and sense modes in the presence of an input angular rate [1]. Conventionally, these modes are realized as single degree of freedom (DOF) dynamic systems, each having its own resonant frequency based on the structural design. Thus, two operational methods can be implemented: mode-matched or modemismatched [2]. Mode-matched operation is realized by setting the drive and sense resonant frequencies to be the same. This allows the output to be increased proportional to the sense mode quality factor, yielding sensors with higher sensitivities at the cost of reduced bandwidth and robustness [3]. Separating the resonant frequencies by some prescribed amount, or mode-mismatching, is more common since the increased robustness (at the cost of sensitivity) is necessary for most practical applications, such as automotive and consumer electronics $[4,5]$.

Previously, a gyroscope design concept intended for robust operation was introduced using an expanded sense mode design space through increased degrees of freedom [6]. Specifically, the device used two coupled sense masses forming a 2-DOF dynamic system with two sense mode resonant frequencies and a wide region of relatively

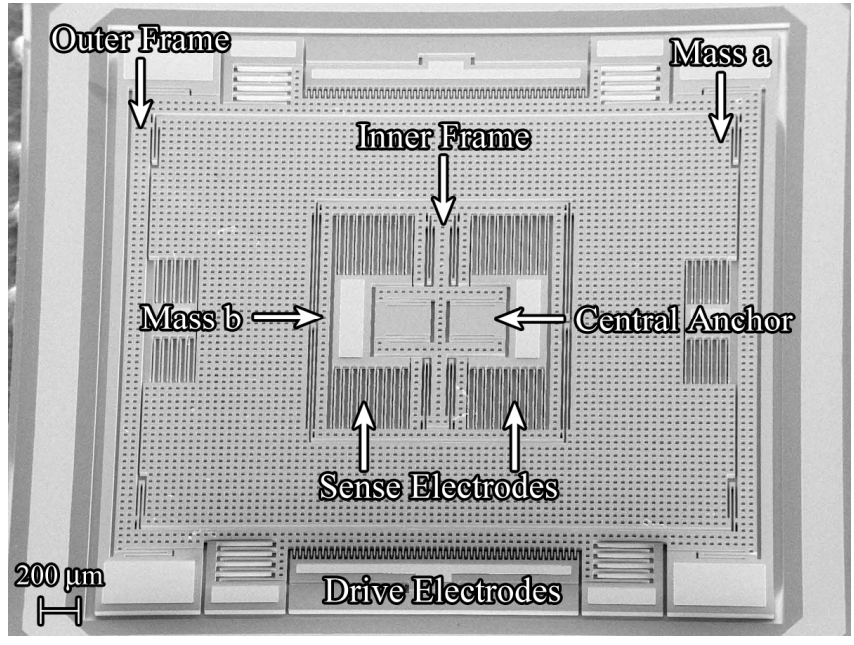

Figure 1: Scanning electron micrograph of a fabricated gyroscope prototype allowing interchangeable operation.

constant amplitude between them. While the gain and the bandwidth of this operational region is controlled solely by the resonant frequency spacing, a constraint dictates a trade-off between minimal achievable spacings, die size, and sense capacitance as the operational frequency of the device is increased [7]. This is due to the use of a dynamic vibration absorber type 2-DOF design, consisting of two masses and only two suspensions, thereby eliminating the ability to independently define the frequency spacing and the coupling between the masses. In contrast, a complete 2-DOF system, consisting of two masses and three suspensions, alleviates the constraint imposed on system parameters as frequency is increased [8].

A z-axis micromachined gyroscope design, Figure 1, was first presented in [9] that takes advantage of a complete 2-DOF sense mode dynamic system allowing the independent adjustment of the sense mode frequency separation and amount of coupling between the masses. Additionally, a new operational method was introduced where the drive mode could be interchangeably placed between the sense mode peaks (robust mode) or mode-matched to one of the 2-DOF sense mode resonant frequencies (precision mode). This paper builds upon previous work by examining the effect of sense mode coupling on the precision and robust mode gains for both atmospheric and vacuum operation. 


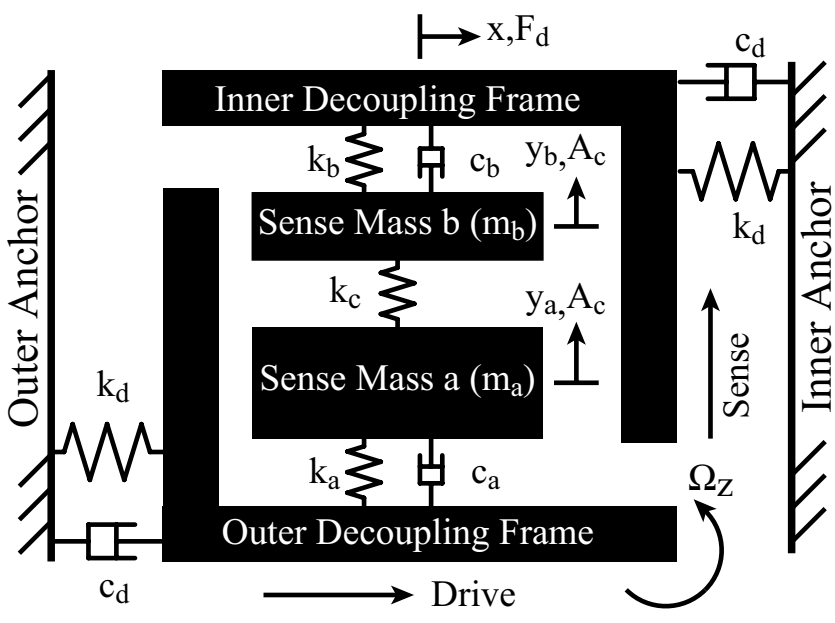

Figure 2: Structural diagram of gyroscope concept with inner and outer frames mechanically decoupled from a complete 2DOF sense mode.

\section{DESIGN CONCEPT}

A structural schematic of the new gyroscope concept is shown in Figure 2. The design, like previous multidegree of freedom sense mode devices, consists of a conventional, 1-DOF drive mode that is mechanically decoupled from a 2-DOF sense mode dynamic system formed by the two coupled sense masses, $\mathrm{m}_{\mathrm{a}}$ and $\mathrm{m}_{\mathrm{b}}$. The major difference between the presented design concept and the previous multi-DOF implementation [6] is the addition of a second, inner decoupling frame that connects the smaller sense mass, $\mathrm{m}_{\mathrm{b}}$, to a central anchor. This acts to preserve $\mathrm{x}-\mathrm{y}$ symmetry while also enabling the inclusion of a third suspension, $\mathrm{k}_{\mathrm{b}}$, into the coupled system. Thus, a complete 2-DOF sense mode, consisting of two masses, $\mathrm{m}_{\mathrm{a}}$ and $\mathrm{m}_{\mathrm{b}}$, and three suspension elements, $\mathrm{k}_{\mathrm{a}}, \mathrm{k}_{\mathrm{b}}$, and $\mathrm{k}_{\mathrm{c}}$, can be realized.

\section{2-DOF Design Equations}

Since the drive mode of the device is a conventional, 1-DOF resonant system, the desired natural frequency can be obtained independent of the sense mode dynamics through adjustment of the total drive mode mass and/or the stiffness; the sense mode, however, is more complex due to the expanded parameter space of the multiple masses. In this case, the structural parameters required to obtain the desired 2-DOF natural frequencies must be determined from the eigenvalue equation. For the coupled system in Figure 2, the solution to the eigenvalue equation yields

$$
{\omega_{1,2}}^{2}=\frac{1}{2}\left[\omega_{a}^{2}+\omega_{b}^{2} \mp \sqrt{\left(\omega_{a}^{2}-\omega_{b}^{2}\right)^{2}+4 \omega_{c}^{4}}\right],
$$

where $\omega_{1,2}$, are the lower and higher desired sense mode resonant frequencies in terms of the structural parameters,

$$
\omega_{a}^{2}=\frac{k_{a}+k_{c}}{m_{a}}, \omega_{b}^{2}=\frac{k_{b}+k_{c}}{m_{b}}, \omega_{c}^{2}=\frac{k_{c}}{\sqrt{m_{a} m_{b}}} .
$$

The terms in equation (2) have structural significance since $\omega_{\mathrm{a}}$ and $\omega_{\mathrm{b}}$ are the uncoupled natural frequencies of mass $\mathrm{m}_{\mathrm{a}}$ and $\mathrm{m}_{\mathrm{b}}$, respectively (assuming the other mass is held fixed), while $\omega_{c}$ expresses the strength of the coupling between the masses.

Design equations can be obtained by solving equation (1) for the structural parameters $\omega_{\mathrm{a}}$ and $\omega_{\mathrm{b}}$ in terms of the desired sense mode natural frequencies $\omega_{1}$ and $\omega_{2}$ :

$$
\omega_{a, b}^{2}=\frac{1}{2}\left[\omega_{1}^{2}+\omega_{2}^{2} \pm \sqrt{\left(\omega_{1}^{2}-\omega_{2}^{2}\right)^{2}-4 \omega_{c}^{4}}\right] .
$$

Thus, the values of $k_{a}$ and $k_{b}$ can be determined using equation (3) once the value of the sense masses (typically a function of outside parameters such as die size, wafer thickness, etc.) and the amount of coupling are known. The coupling, however, cannot be arbitrarily defined, but rather must be chosen so that equation (3) results in physically realizable parameters. Specifically, this requires that

$$
0<\omega_{c}^{4} \leq \frac{1}{4}\left(\omega_{1}^{2}-\omega_{2}^{2}\right)^{2},
$$

which defines the range of allowable coupling stiffnesses up to a maximum determined by the desired sense mode resonant frequencies. Equation (4) can be expressed as a fraction of allowable coupling, $\omega_{\mathrm{c}}{ }^{2}=1 / 2 \varepsilon\left(\omega_{1}{ }^{2}-\omega_{2}{ }^{2}\right)$, so that equation (3) becomes,

$$
\omega_{a, b}^{2}=\frac{1}{2}\left[\omega_{1}^{2}+\omega_{2}^{2} \pm\left(\omega_{1}^{2}-\omega_{2}^{2}\right) \sqrt{1-\varepsilon^{2}}\right],
$$

where $\varepsilon$ is the coupling parameter and is defined in the range $0<\varepsilon \leq 1$.

\section{INTERCHANGEABLE OPERATION}

The complete 2-DOF sense mode provides distinct advantages over previous multi-DOF sense systems, such as the elimination of operational frequency scaling trade-offs [7], as well as the ability to independently adjust the amount of coupling between the masses for a given frequency spacing. The complete 2-DOF sense mode also enables a new operational method for multi-DOF gyroscopes: interchangeable operation in both precision and robust modes in a single device.

The concept of interchangeable operation is shown in Figure 3, which presents a conceptual 2-DOF frequency response highlighting the robust and precision regions. Similar to previous multi-DOF devices, the robust mode corresponds to operation between the sense mode resonant frequencies, providing a wide region of relatively constant gain and phase. Precision operation, however, consists of mode-matching the drive to one of the sense mode resonant peaks. Much like conventional devices, precision operation takes advantage of sense mode resonant gain, allowing increasingly larger sensitivities through operation in vacuum. Also, the complete 2-DOF system can be designed to provide a precision mode gain advantage over similar 1-DOF systems for equivalent damping conditions. 


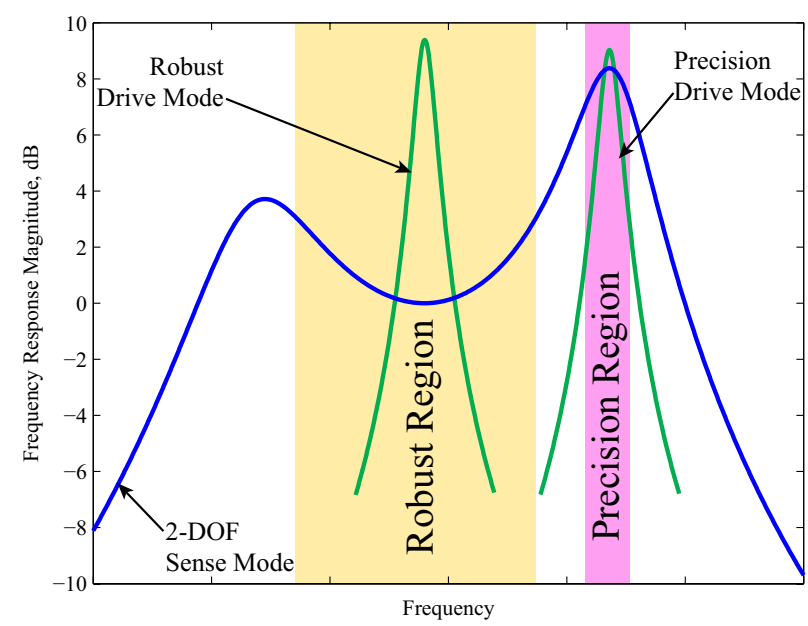

Figure 3: Conceptual frequency response of complete 2-DOF sense mode highlighting the robust and precision regions as well as the corresponding drive mode positions.

The gains of the robust and precision modes are independently adjustable, allowing both to be tailored to the specific application. The robust mode gain is determined at the design level through the specification of the sense mode frequency separation and is generally unaffected by changes in pressure $[6,7]$. The precision mode gain, however, is dependent on both the coupling stiffness as well as the pressure in which the device is operated. Therefore, the gain advantage of precision versus robust operation in atmospheric pressure is set by mechanical design; the precision mode gain improvement is further increased by operation in vacuum.

\section{FABRICATION}

The devices used for the experimental characterization presented below were fabricated using an in-house, two mask, wafer scale silicon-on-insulator (SOI) process with a conductive $50 \mu \mathrm{m}$ device layer and a $5 \mu \mathrm{m}$ buried oxide. First, a front side metalization process was used to define bonding pads via lift-off followed by a Deep Reactive Ion Etching (DRIE) step to define the sensors in the SOI device layer. The minimum feature size of the overall process was $5 \mu \mathrm{m}$ which was used to define the capacitive gaps. The perforated structures were released using a timed HF etch followed by dicing, packaging, and wire bonding. An SEM image of a fabricated gyroscope prototype is presented in Figure 1.

\section{DESIGN TRADE-OFFS}

In order to determine the effects of coupling stiffness on the robust to precision mode gain ratio, simulated frequency responses were performed for complete 2-DOF systems in air with constant sense mode frequency spacings while varying the amount of coupling. As shown in Figure 4, the robust mode gains for all coupling amounts

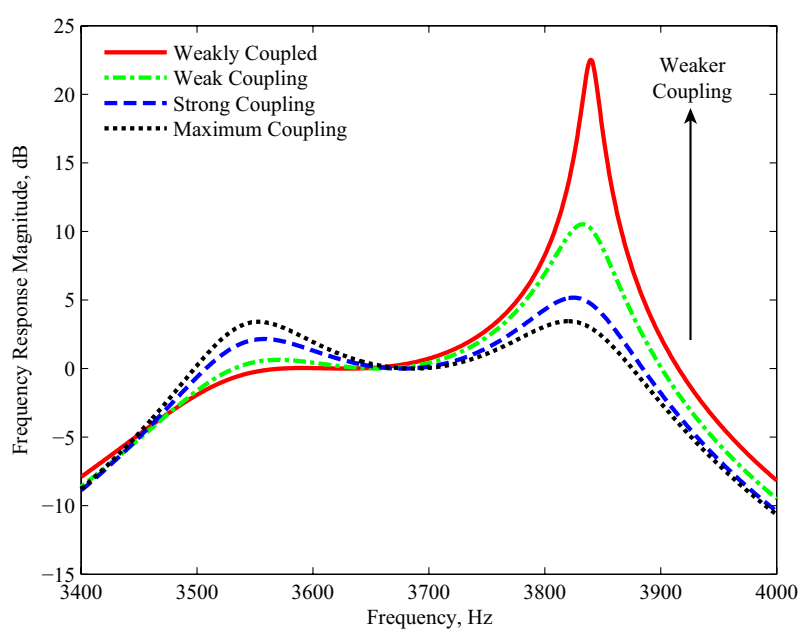

Figure 4: Simulated sense mode frequency responses for various coupling amounts revealing the trade-off: weaker coupling results in larger precision mode gain for atmospheric pressure.
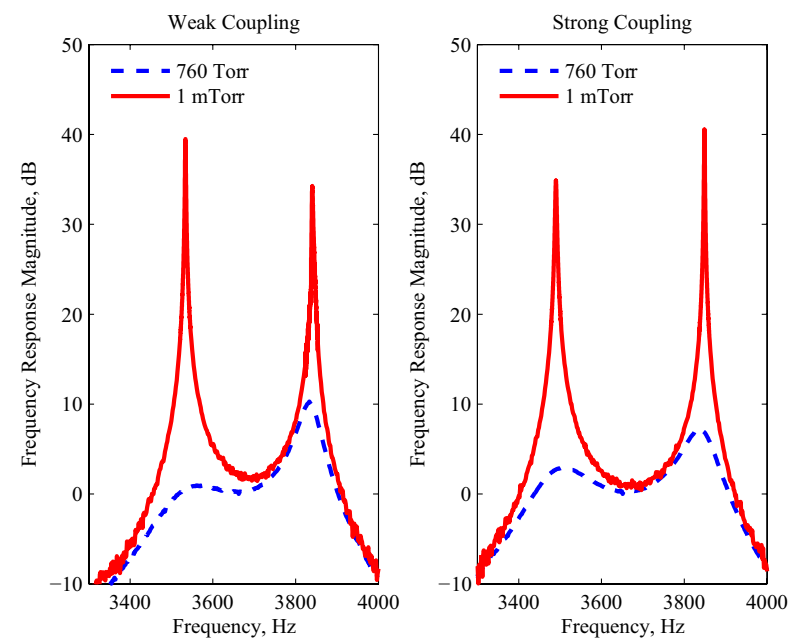

Figure 5: Experimental frequency responses in air and vacuum demonstrating that stronger coupling is desirable due to the larger precision mode gain and the unaffected robust mode.

are essentially the same; the bandwidth, however, increases with weaker coupling do to the inclusion of the lower in-phase peak. The precision mode gains, on the other hand, change drastically with coupling starting at minimal values for maximum $(\varepsilon=1)$ and strong coupling $(0.9 \leq \varepsilon<$ 1). Larger precision mode gain improvement for atmospheric operation is obtained with decreasing coupling stiffness resulting in an increase of more than $20 \mathrm{~dB}$ versus the robust mode for weakly coupled systems $(\varepsilon<0.3)$. Thus, weak coupling is ideal for purely atmospheric operation as it maximizes the precision mode gain improvement.

In order to verify the simulations and to determine the effect of coupling stiffness on the robust and precision mode gains in vacuum, several sense mode systems were designed with iterated coupling amounts and fabricated 


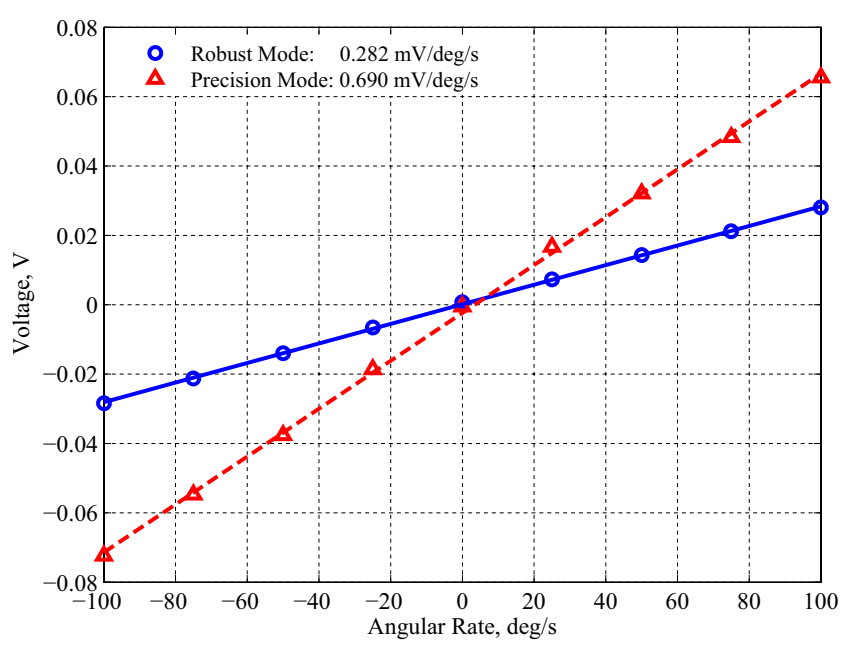

Figure 6: Experimental angular rate characterization for both operational modes in air revealing a 2.4 times improvement in scale factor for the precision mode.

using the process described above. Figure 5 presents experimental frequency response plots for strong and weak coupling stiffness sense mode systems in both air and 1 mTorr vacuum. As expected, the weak coupling system provides a higher precision mode gain improvement in air (10 dB versus $7 \mathrm{~dB}$ ) versus the $240 \mathrm{~Hz}$ wide robust gain regions. On the other hand, the strong coupling stiffness system in vacuum provides the larger precision mode improvement of more than $40 \mathrm{~dB}$ versus the robust region. Additionally, the robust region of the strong coupling stiffness system is unaffected as the pressure is decreased; for the weak stiffness system, however, this is not the case since the robust region contained the lower, in-phase peak in air, which changes dramatically in vacuum.

To verify the rate operation of both robust and precision modes, a device with strong coupling was characterized in atmospheric pressure at constant angular rates, Figure 6. For robust operation, the experimentally obtained scale factor was $0.282 \mathrm{mV} / \mathrm{deg} / \mathrm{s}$ while for precision operation it was $0.690 \mathrm{mV} / \mathrm{deg} / \mathrm{s}$. This 2.4 times improvement for precision mode operation in air compares to the predicted increase of the strong coupling stiffness system from Figure 5; for operation in vacuum, however, the precision mode scale factor is expected to improve over 40 $\mathrm{dB}$ versus the robust mode.

\section{CONCLUSION}

In conclusion, design trade-offs were presented for a gyroscope design allowing interchangeable operation in precision or robust modes. For atmospheric pressure, weakly coupled systems are desirable due to the larger achievable precision mode gain improvements (over 20 $\mathrm{dB}$ ); for vacuum operation, however, the strong coupling system resulted in the larger precision mode gain (over 40 $\mathrm{dB}$ ) while leaving the robust region relatively unaffected.

\section{ACKNOWLEDGEMENTS}

This work was supported by the National Science Foundation (CMS-0409923), Systron Donner Automotive (BEI-36974), the UC Discovery program (ELE04-10202), and the Naval Surface Warfare Center (N00178-08C1014). The devices were designed, modeled, and characterized in the MicroSystems Laboratory and fabricated in the Integrated Nanosystems Research Facility at the University of California, Irvine. The authors thank the Carl Zeiss Center of Excellence for use of the Ultra 55 SEM.

\section{REFERENCES}

[1] A. M. Shkel, "Type I and Type II Micromachined Vibratory Gyroscopes," in Proc. of IEEE/ION PLANS, San Diego, CA, USA, April 24-27, 2006.

[2] M. S. Weinberg and A. Kourepenis, "Error Sources in In-Plane Silicon Tuning-Fork MEMS Gyroscopes," $J$. Microelectromech. Syst., vol. 15, no. 3, pp. 479-491, June 2006.

[3] N. Yazdi, F. Ayazi, and K. Najafi, "Micromachined Inertial Sensors," Proc. IEEE, vol. 86, no. 8, pp. 16401659, August 1998.

[4] R. Neul, U. M. Gomez, K. Kehr, W. Bauer, J. Classen, C. Doring, E. Esch, S. Gotz, J. Hauer, B. Kuhlmann, C. Lang, M. Veith, and R. Willig, "Micromachined Angular Rate Sensors for Automotive Applications," IEEE Sensors J., vol. 7, no. 2, pp. 302-309, February 2007.

[5] J. A. Geen, S. J. Sherman, J. F. Chang, and S. R. Lewis, "Single-Chip Surface Micromachined Integrated Gyroscope with $50 \mathrm{deg} / \mathrm{h}$ Allan Deviation," IEEE J. Solid-State Circuits, vol. 37, no. 12, pp. 1860-1866, December 2002.

[6] C. Acar and A. M. Shkel, "Inherently Robust Micromachined Gyroscopes with 2-DOF Sense-Mode Oscillator," J. Microelectromech. Syst., vol. 15, no. 2, pp. 380-387, April 2006.

[7] A. R. Schofield, A. A. Trusov, and A. M. Shkel, "Effects of Operational Frequency Scaling in MultiDegree of Freedom MEMS Gyroscopes," IEEE Sensors $J$., vol. 8, no. 10, pp. 1672-1680, October 2008.

[8] A. A. Trusov, A. R. Schofield, and A. M. Shkel, "Performance Characterization of a New TemperatureRobust Gain-Bandwidth Improved MEMS Gyroscope Operated in Air," Sensors and Actuators A: Physical, In Press, 2008.

[9] A. R. Schofield, A. A. Trusov, and A. M. Shkel, "Micromachined Gyroscope Design Allowing for Both Robust Wide-Bandwidth and Precision Mode-Matched Operation," in Proc. IEEE Sensors, Lecce, Italy, October 26-29, 2008.

\section{CONTACT}

*A.R. Schofield, +1-949-824-6314; aschofie@uci.edu 